\title{
Bias in MRI Measurements of Apparent Diffusion Coefficient and Kurtosis: Implications for Choice of Maximum Diffusion Encoding
}

\section{Nima Gilani ${ }^{1}$ (D) $\cdot$ Glyn Johnson ${ }^{2}$}

Received: 4 May 2018 / Revised: 25 June 2018 / Published online: 12 August 2018

(c) The Author(s) 2018

\begin{abstract}
Tissue water diffusion is non-Gaussian and the expressions used to calculate diffusion parameters are approximations which introduce systematic errors dependent on the maximum diffusion encoding, diffusion time, etc. This study aimed at characterizing biases in estimates of both apparent diffusion coefficient and kurtosis, and determines their dependence on these parameters. Similar to the approach of several previous studies, Taylor expansion of the diffusion signal was used to calculate biases. Predicted errors were compared with data from one volunteer. Predicted errors agreed well with the measured errors and also the published diffusion tensor imaging measurements. The equations derived predict biases in measured diffusion parameters and explain much of the discrepancy between measurements obtained with different acquisition protocols. The equations may also be used to choose appropriate diffusion encoding for diffusion weighted, tensor, and kurtosis imaging.
\end{abstract}

\section{Introduction}

Quantitative diffusion magnetic resonance imaging (MRI) has proven useful in characterizing damaged tissue in a number of different diseases such as breast cancer [1-3], prostate cancer [4,5], multiple sclerosis [6], etc. due to the effect of changes in cell density or morphology, and tissue composition on water diffusion.

In general, the signal generated in a diffusion-weighted MRI sequence is given by $[7,8]$

$$
S=S_{0} e^{a_{1} b D+a_{2} b^{2} D^{2}+a_{3} b^{3} D^{3}+\cdots},
$$

Glyn Johnson

glyn.johnson@uea.ac.uk

1 Department of Cognitive Neuroscience, Faculty of Psychology and Neuroscience, Maastricht University, Maastricht, The Netherlands

2 Norwich Medical School, University of East Anglia, Bob Champion Research and Educational Building, James Watson Road, Norwich Research Park, Norwich NR4 7UQ, UK 
where $S_{0}$ is the signal in the absence of diffusion weighting, $a_{i}$ are a series of numerical coefficients, $D$, is the diffusion coefficient, and $b$ is the diffusion encoding parameter which for Stejskal-Tanner pulses is given by [9]

$$
b=\gamma^{2} \delta^{2} g^{2}\left(\Delta-\frac{\delta}{3}\right)
$$

where $\gamma$ is the gyromagnetic ratio, $\delta$ is the length of the diffusion weighting gradient pulses, $\Delta$ is the time interval between the leading edge of the pulses, and $g$ is the amplitude of the pulses.

The coefficients, $a_{i}$, depend on the shape of the probability density function, $P(r)$, describing diffusional displacement. In bulk fluids, $P$ is Gaussian, $a_{1}=-1$, and $a_{i>1}=0$, leading to the familiar equation:

$$
S=S_{0} \mathrm{e}^{-b D} .
$$

It has been recognised that diffusion in tissue is non-Gaussian due to compartmentalization, restriction, and hindrance of water. Hence, Eq. (3) is only approximate and the signal can be more accurately described by $[10,11]$

$$
S=S_{0} \mathrm{e}^{-b D+\frac{K b^{2} D^{2}}{6}+O\left(b^{3} D^{3}\right)},
$$

where $K$ is the kurtosis of $P$, i.e., the normalized fourth moment [10, 11]:

$$
K=\frac{\int r^{4} P(r) \mathrm{d} r}{\left(\int r^{2} P(r) \mathrm{d} r\right)^{2}}-3=\frac{\left\langle(\text { n.s })^{4}\right\rangle}{\left\langle(\mathbf{n . s})^{2}\right\rangle^{2}}-3,
$$

where $\mathbf{s}$ is net displacement vector, and $\mathbf{n}$ is the unit vector in the direction of interest.

Diffusion parameters are measured by acquiring images at multiple different diffusion encoding $b$ values and fitting the model to these signals using non-linear least squares algorithms. Errors in estimates are due both to thermal noise in the signal and to biases due to failure to take the higher order terms of Eq. (1) into account. As a rule of thumb, errors in estimates of parameter $X$ (either $D$ or $K$ ) due to thermal noise are minimized using a maximum $b$ value, $b_{\max }$, that maximizes $\mathrm{d} S / \mathrm{d} X[12,13]$. Minimizing thermal noise in simple estimates of $D$ using Eq. (3) requires $b_{\max } \sim 1 / D$. To measure kurtosis, larger values $\left(b_{\max }>2 / D\right)$ are usually recommended [12]. However, as $b_{\max }$ increases, bias in estimates increases due to the increasing influence of higher order terms in Eq. (1). It has previously been recommended that for kurtosis imaging $b_{\max }$ should be less than $3 / D K[10,11]$, the point at which signals described by Eq. (4) no longer monotonically decrease.

Furthermore, there are numerous reports in the radiology literature on the effect of $b$ value choice on diffusion estimates (e.g., [14-16]) but with little attempt at explanation.

In this report, we derive analytical expressions for the bias in estimates of $D$ using Eq. (3) and in $D$ and $K$ using Eq. (4); this approach is similar to a recent study by 
Chuhutin et al. [17]. These expressions allow better assessment of the trade-off between errors due to thermal noise and estimation bias. Predicted biases are compared with results from a recently published diffusion tensor imaging (DTI) study of the brain [18].

\section{Theory}

\subsection{Diffusion Measurement Bias}

For simplicity, we will consider only the simplest diffusion measurement using two $b$ values, $b_{\min }$ and $b_{\max }$. Our estimate of $D$ is then given by

$$
\tilde{D}=-\frac{\operatorname{In}\left[S\left(b_{\max }\right) / S\left(b_{\min }\right)\right]}{b_{\max }-b_{\min }} \approx D-\frac{K D^{2}\left(b_{\max }+b_{\min }\right)}{6}
$$

where we have assumed that the higher order terms of Eq. (1) are dominated by the kurtosis term. Fractional error caused using very high maximum $b$ values then given by

$$
e_{D}=\left|\frac{\tilde{D}-D}{D}\right|=\frac{D K\left(b_{\max }+b_{\min }\right)}{6}
$$

\subsection{Diffusion Kurtosis Measurement Bias}

Calculation of the bias in kurtosis measurements requires calculation of the third order term in Eq. (1), corresponding the sixth moment of $P$. Following the approach of Jensen et al. [10, 19], we can derive the following expression for the sixth moment, $L$ [20] (details are given in the "Appendix"):

$$
L=\frac{\left\langle(\text { n.s })^{6}\right\rangle}{\left\langle(\text { n.s })^{2}\right\rangle^{3}}-15 \frac{\left\langle(\text { n.s })^{4}\right\rangle}{\left\langle(\text { n.s })^{2}\right\rangle^{2}}+30
$$

where again $\mathbf{s}$ is net displacement vector, and $\mathbf{n}$ is the unit vector in the direction of interest. We refer to $L$ as the ektasis after the Greek for stretching out or extension (kurtosis is derived from the Greek for bulging). Including ektasis in the Taylor expansion $[10,21,22]$ of the diffusion signal gives

$$
\ln \left(S(b) / S_{0}\right)=-b D+\frac{b^{2} D^{2} K}{6}+\frac{b^{3} D^{3} L}{90}+O\left(b^{4} D^{4}\right),
$$

where the additional term has been derived by a simple extension of the method given in the appendix of Ref. [10].

For free (i.e., Gaussian) diffusion, $L$, like $K$, is zero. For a box-car distribution corresponding to diffusion in an impermeable cavity with small radius compared with the 
diffusion distance, $L$ is $48 / 7$. By comparison, $D=r^{2} / 6 T_{\mathrm{TD}}$, where $r$ is the radius of the cavity and $T_{\mathrm{TD}}$ is the total diffusion time, and $K=-1.2$ [23] (the derivation of these values are given in the "Appendix").

To calculate errors in estimates of $D$ and $K$, we make the simplifying assumptions that only three $b$ values are used: $0, b_{\max } / 2$ and $b_{\max }$. It can be shown that $D$ and $K$ can be estimated by the equations [11]:

$$
\begin{array}{r}
\tilde{D}=2 Q_{1}-Q_{2} \\
\tilde{K}=12 \frac{Q_{1}-Q_{2}}{b_{\max } \tilde{D}^{2}},
\end{array}
$$

where

$$
\begin{aligned}
Q_{1} & =2 \frac{\ln \left(S_{0} / S_{1}\right)}{b_{\max }} \\
Q_{2} & =\frac{\ln \left(S_{0} / S_{2}\right)}{b_{\max }},
\end{aligned}
$$

and $S_{1}$ and $S_{2}$ are the signals acquired with $b=b_{\max } / 2$ and $b=b_{\max }$, respectively. Following the same procedure as before gives (derivation of $\tilde{D}$ and $\tilde{K}$ is in the "Appendix"):

$$
\begin{array}{r}
e_{\mathrm{D}}=\left|\frac{\tilde{D}-D}{D}\right|=\left|\frac{b_{\max }^{2} D^{2} L}{180}\right| \\
e_{\mathrm{K}}=\left|\frac{\tilde{K}-K}{K}\right|=\frac{1}{\left(1+e_{\mathrm{D}}\right)^{2}}\left(\left|\frac{b_{\max } D L}{10 K}\right|+1\right)-1 .
\end{array}
$$

\subsection{Extension to More Complex Acquisitions}

These expressions allow an estimate of the maximum $b$ value to use to avoid bias of greater than a specified fraction for simple measurements of single isotropic compartments. It is straightforward to extend the results to more complex situations.

\subsubsection{Biexponential Diffusion}

The prostate appears to consist of two slowly exchanging compartments, probably the fluid filled ducts and surrounding cellular tissue [4], and thus generates a biexponential diffusion signal $[24,25]$ which is the sum of the signals from each of these compartments. For simple Gaussian measurements, the error in ADC estimate is proportional to $D K$ [Eq. (7)]. Therefore, setting the maximum $b$ value 
to limit measurement error in the compartment with the larger value of $D K$ will automatically also limit measurement error in the other compartment. A similar approach can be taken with non-Gaussian measurements, with the further complication that fractional errors in estimates of $D$ and $K$ are different. If ADC is primarily of interest, maximum $b$ value should be based on the compartment with the largest value of $D^{2} L$ [Eq. (12)]. Conversely, if kurtosis is of most interest, maximum $b$ value should be set based on the compartment with higher values of $D L / K$.

\subsubsection{Non-isotropic Diffusion}

For organs with high diffusion anisotropy, such as white matter multiple directions of diffusion weighting are required [26]. Maximum $b$ value should be chosen on the basis of the highest directional $D K$ (for Gaussian measurements), or $D L$ or $D^{2} L$ (for non-Gaussian measurements). Since axonal direction is not known a priori, it is necessary to apply this value in all gradient directions. This may be of particular importance in estimates rooted in diffusion models [27, 28], since different biases in directional estimates can lead to incorrect estimates of derived parameters such as axonal density, etc.

\section{Methods}

\subsection{Imaging}

This study was approved by the institutional review board of Tehran University of Medical Sciences. To verify Eqs. (7) and (12), a healthy volunteer underwent a multi $b$ value double echo-planar diffusion-weighted imaging (DWI) brain scan using a 3 T Prisma scanner (Siemens Healthcare, Erlangen, Germany), with a 64-channel head coil. Nine $b$ values were used $(0,150,500,700,1000,1500,2000,2500$, and $3000 \mathrm{smm}^{-2}$ ). Other acquisition parameters were: number of slices, 68; diffusion directions, 30; FOV, $256 \times 256 \mathrm{~mm}^{2}$; voxel size, $2 \times 2 \times 2 \mathrm{~mm}^{3}$; TR/TE, 9000/90 ms.

\subsection{Verification of DKI biases}

To obtain predicted errors for diffusion kurtosis imaging (DKI) estimates, similar to those obtained for DWI, it is necessary to measure ektasis. However, such measurements require acquisitions with very high $b$ values (i.e., $>3000 \mathrm{smm}^{-2}$ ) and hence low signal-to-noise ratios (SNRs). Estimates of L were obtained by non-linear least squares fitting of Eq. (9) on all of the $b$ values employed. This process was applied to multiple brain voxels and measured fractional errors in $D$ and $K$ were calculated as

$$
\begin{aligned}
& \frac{D-\tilde{D}}{D} \\
& \frac{K-\tilde{K}}{K} .
\end{aligned}
$$


These measured fractional errors were compared with values predicted from Eq. (12).

In addition, we compared DKI estimates obtained with two different sets of $b$ values: 150,1000 , and $2000 \mathrm{smm}^{-2}$, and 150,1500 , and $3000 \mathrm{smm}^{-2} . D$ and $K$ were derived for each set and plotted against each other. The slope of the regression lines

$$
\begin{aligned}
& D_{150,1500,3000}=k_{\mathrm{D}} D_{150,1000,2000} \\
& K_{150,1500,3000}=k_{\mathrm{K}} K_{150,1000,2000},
\end{aligned}
$$

where the subscripts to $D$ and $K$ refer to the set of $b$ values used for the estimate, were then found.

\subsection{Tissue-Specific $b$ Value Selection}

Similar to [17], the dependency of diffusion estimates on maximum $b$ values was investigated separately for the white and grey matters. For this aim, $e_{\mathrm{D}}$ and $e_{\mathrm{K}}$ of 200 voxels containing white matter or grey matter voxels were averaged for maximum $b$ values of 1500, 2000, and $3000 \mathrm{smm}^{-2}$.

\section{Results}

\subsection{Theoretical predictions}

Figure 1 gives plots of the fractional error in diffusion estimates calculated using Eq. (7) vs. the dimensionless quantity $D . b_{\max }$ with $b_{\min }=0$. Even at moderate values of kurtosis, bias in estimates of $D$ are $\sim 10 \%$ when $b_{\max }=D$. Figure 2 gives plots in the fractional error of both $D$ and $K$ calculated using Eq. (12) vs. D. $b_{\max }$. The bias in estimates of $D$ is much reduced in diffusion kurtosis imaging to diffusion weighted imaging, even for higher values of $b_{\max }$. However, bias in estimates of $K$ itself is large for even for low values of ektasis.

\subsection{DKI}

Figure 3 gives plots of measured fractional errors vs. fractional errors predicted by Eq. (12) for $D$ (Fig. 3a) and $K$ (Fig. 3b). The correlation coefficients between measured and predicted errors were 0.9660 and 0.9344 for $D$ and $K$, respectively.

Figure 4 shows plots of $D$ (a) and $K$ (b) derived using $b$ values of 150,1500 , and $3000 \mathrm{smm}^{-2}$ against those derived using $b$ values of 150,1000 , and $2000 \mathrm{smm}^{-2}$. The slopes of the regression lines, $k_{\mathrm{D}}$ and $k_{\mathrm{K}}$ [Eq. (14)] were 0.87 , and 0.85 , respectively. Both values are less than one in agreement with the theoretical predictions of Eq. (12). 


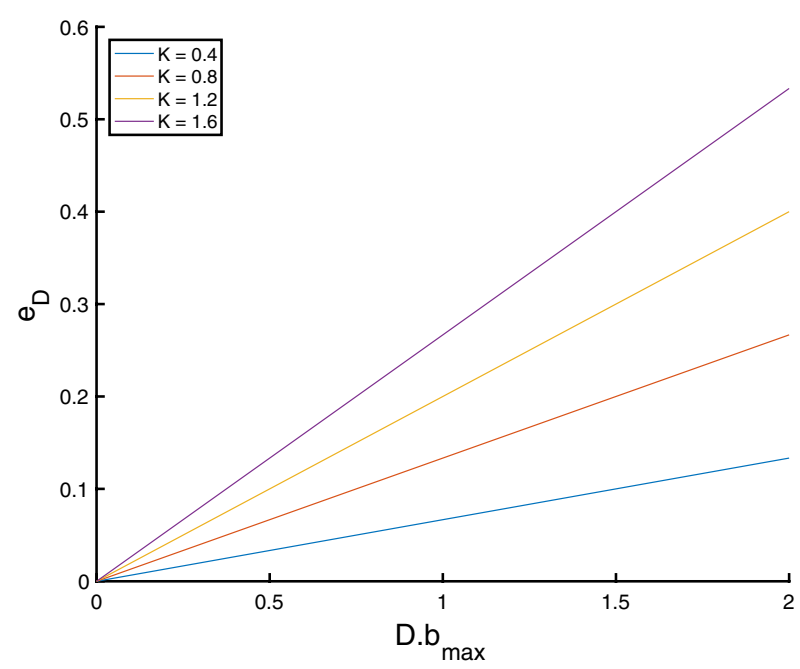

Fig. 1 Plot of fractional error in ADC estimates, $e_{\mathrm{D}}$, vs. D. $b_{\max }$, calculated using Eq. (7) with $b_{\min }=0$ and four values of kurtosis, $K$

\subsection{DKI Biases for WM and GM}

Measured $\left(e_{\mathrm{D}}, e_{\mathrm{K}}\right)$ in grey matter were $(0.06,0.23),(0.07,0.27)$, and $(0.12,0.36)$ for maximum $b$ values of 1500,2000 , and $3000 \mathrm{smm}^{-2}$, respectively. In comparison measured $\left(e_{\mathrm{D}}, e_{\mathrm{D}}\right)$ for white matter were $(0.02,0.16),(0.03,0.21)$, and $(0.07,0.28)$ for maximum $b$ values of 1500,2000 , and $3000 \mathrm{smm}^{-2}$, respectively. In addition, ADC and kurtosis of a single slice at two different maximum $b$ values, and the corresponding $e_{\mathrm{D}}, e_{\mathrm{D}}$ for maximum $b$ value of $3000 \mathrm{smm}^{-2}$ are shown in Fig. 5.

\section{Discussion}

There are numerous reports in the radiology literature of the $b$ value dependence of diffusion estimates (e.g., [14-16, 29-33]). Such protocol dependencies complicate the comparison of results from different institutions and hinder establishment of reliable diagnostic thresholds. Although standardization is one means of ensuring consistency, this is difficult to achieve, since researchers and clinicians are often reluctant to modify protocols with which they have become familiar, and trust. Furthermore, there are often differing restrictions on parameter selection imposed by different scanner manufacturers. In this study, we demonstrate that much of the $b$ value dependence is due to the failure to take into account high order terms in the equation used to derive diffusion parameters.

Lanzafame et al. [18] had already observed biases in estimation of axonal, radial, and mean diffusivity with increasing the maximum $b$ values. The theoretical predictions presented here are in good agreement with their findings. In addition, the results are in agreement with the conclusions of [34] that decreasing maximum $b$ 

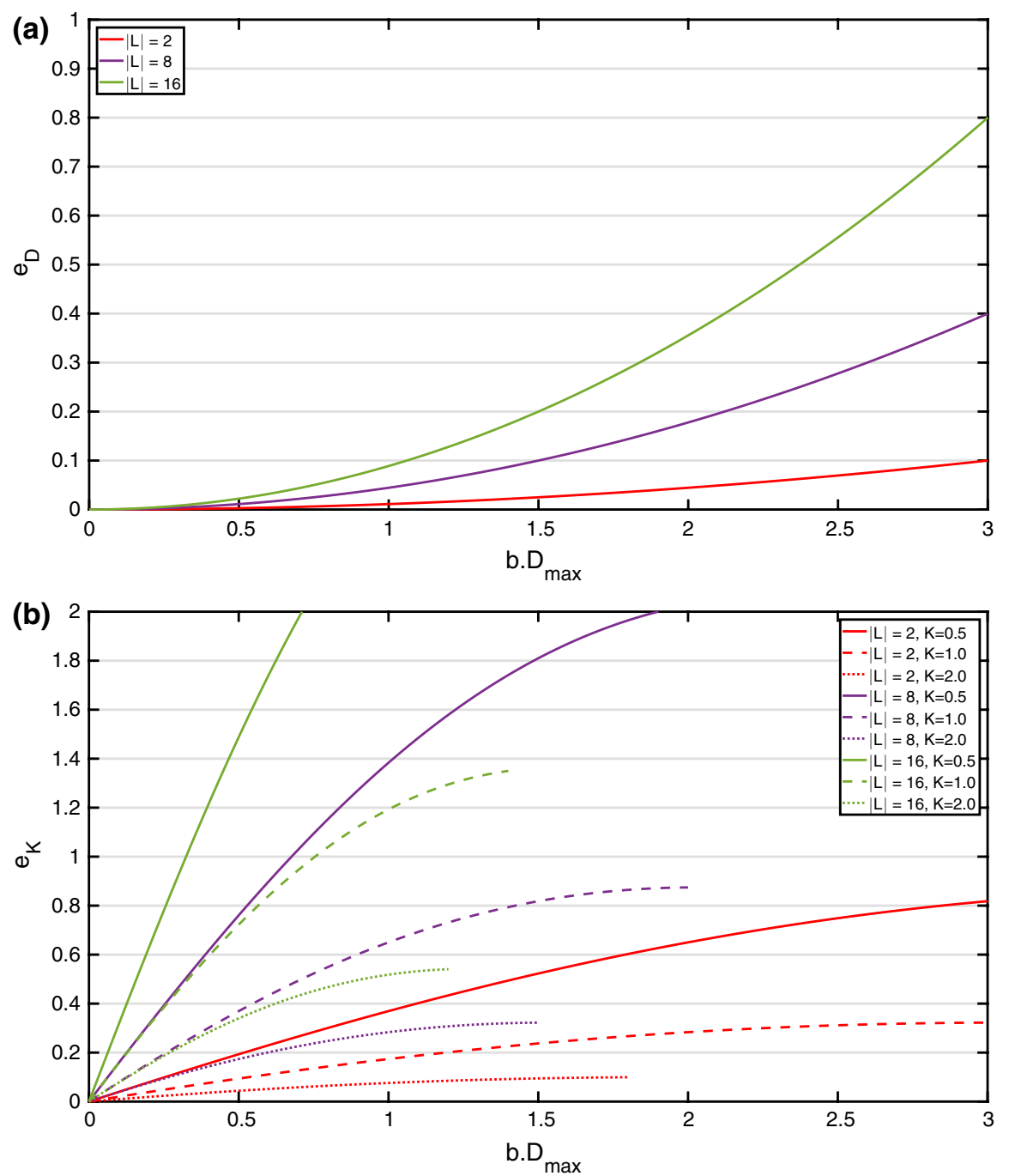

Fig. 2 Plot of fractional error in $\mathbf{a} \mathrm{ADC}$ and $\mathbf{b}$ kurtosis estimates, $e_{\mathrm{D}}$ and $e_{\mathrm{K}}$, vs. D. $b_{\max }$, calculated using Eq. (12) with $b_{\min }=0$, three values of ektasis, $L(2,8$, and 16), and three values of $K(0.5,1$, and 2$)$. Note: $e_{\mathrm{D}}$ is independent of $\mathrm{K}$

values results in more accurate estimation of diffusion kurtosis parameters at the cost of additional random noise.

Chuhutin et al. [17] recommend reasonable maximum $b$ values of $2500 \mathrm{smm}^{-2}$ for measuring DKI parameters in the white matter; however, their recommended maximum $b$ values for grey matter are too small if the effect of noise on parameter estimation is considered. This is because using higher $b$ values are necessary to get precise parameter estimates in diffusion kurtosis imaging [12]. Hence, finding the trade-off between accuracy (the systematic estimate biases) and increased precision 

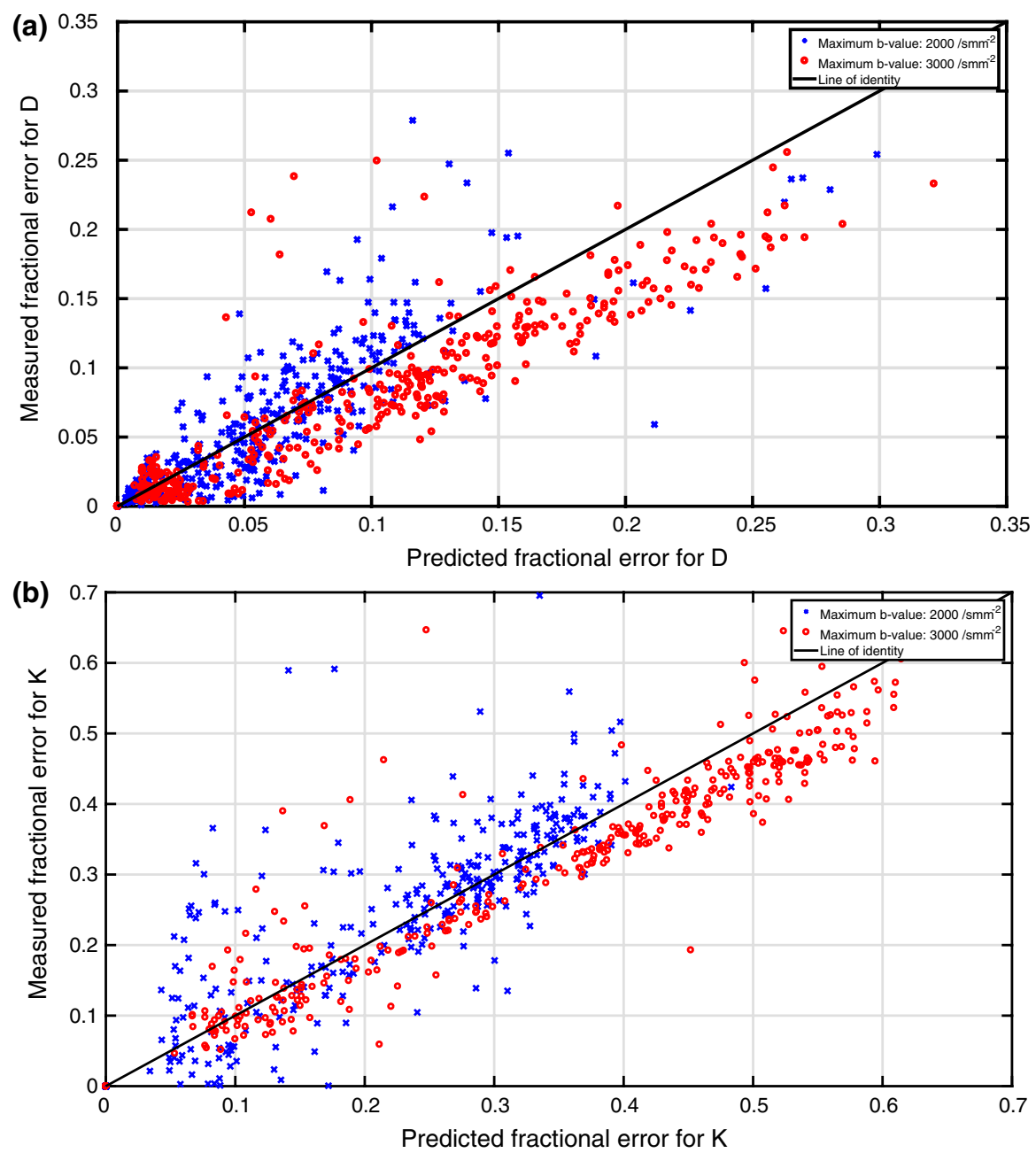

Fig. 3 Measured fractional errors [Eq. (13)] vs. fractional errors predicted by Eq. (12) for a $D$ and $\mathbf{b} K$. The black solid line is the line of identity

(using higher maximum $b$ values) remains an open question for each study depending on the tissue type and protocol details.

There are a variety of sources of variation in diffusion estimates. First, measured ADC depends on diffusion time (e.g., [4]), signal-to-noise ratio of the scanner, and tissue type. In this study, we have shown one of the most important sources of variation is the choice of maximum $b$ value and also how these sources error are dependent on each other. In other words, the choice of maximum $b$ values for any tissue type, region, or organ of interest should consider (a) its $e_{\mathrm{D}}, e_{\mathrm{K}}$ for different $b$ values and diffusion times and (b) the optimization tables in [12] that formulate $D$ and $K$ estimation errors due to thermal noises which are dependent on signal-to-noise 
(a)

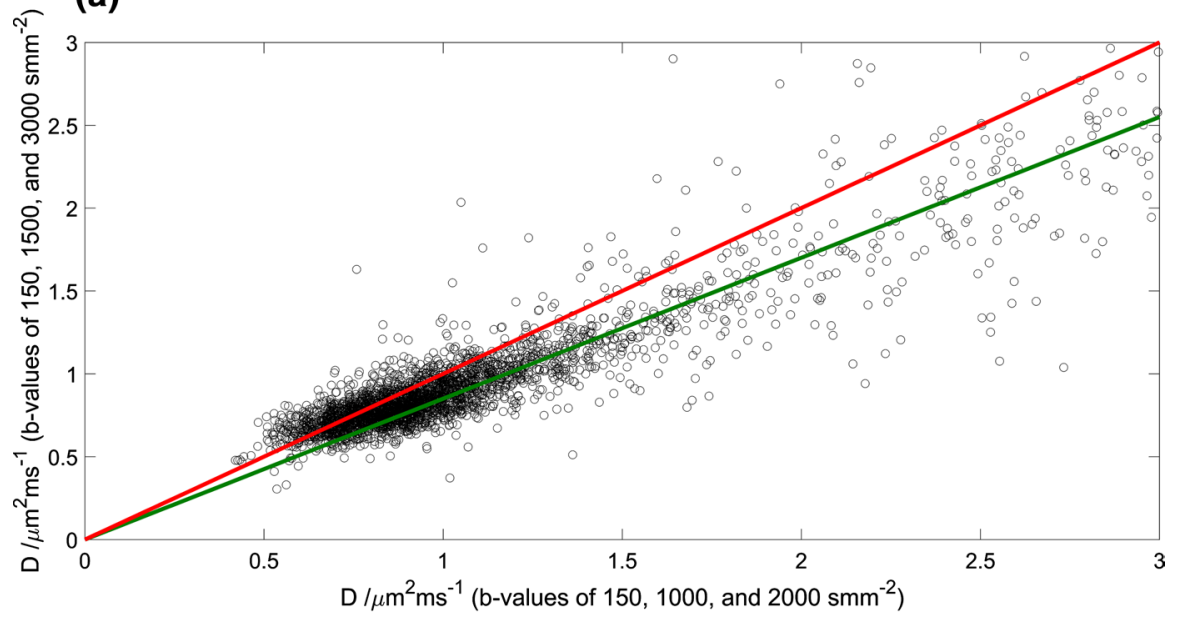

(b)

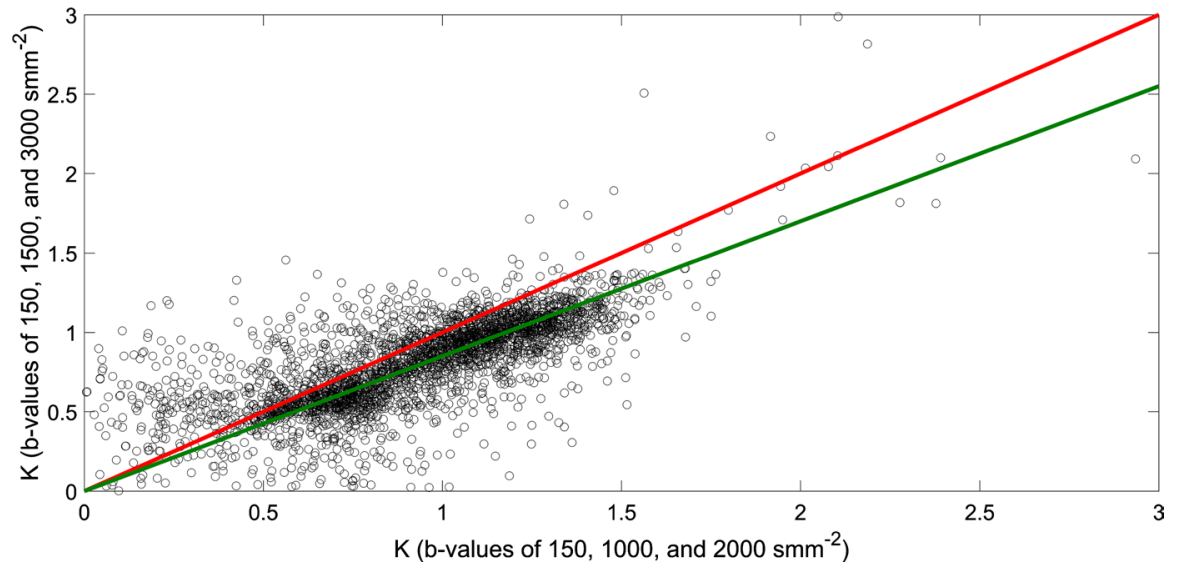

Fig. 4 Plot of parameter estimates obtained with $b$ values of 150, 1500, and 3000, vs. those obtained with $b$ values of 150,1000 , and $2000 \mathrm{smm}^{-2}$ for $\mathbf{a} D$ and $\mathbf{b} K$ in the brain of a volunteer. The red and green lines are the lines of identity and regression, respectively (color figure online)

ratios. Second, the value of the minimum $b$ value will determine the contribution of microvascular signal through the IVIM effect.

In addition, for tissues with biexponential diffusion signal such as the prostate $[4,5]$, separate consideration of biases for each compartment might help in better modelling and estimation of their water fractions. Such knowledge might also help in improving multi-exponential models aiming at characterization of diffusion at boundaries of white matter, grey matter, or cerebrospinal fluids [28].

There are several limitations to this study. First, only the simplest two and three $b$ value acquisitions were considered. Biases in over-determined, multiple $b$ value acquisitions are less amenable to analysis. This problem might be addressed with 
(a)

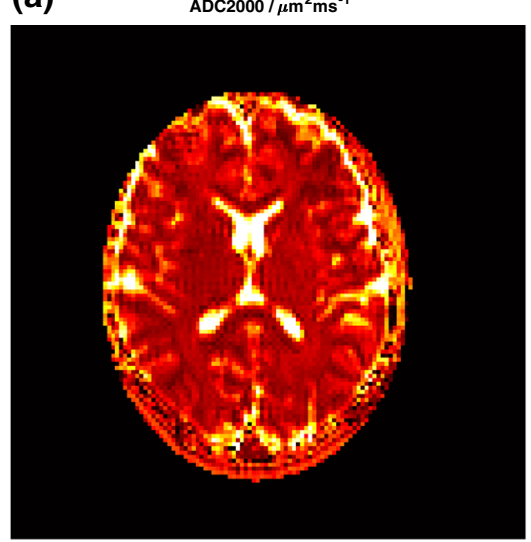

(c)

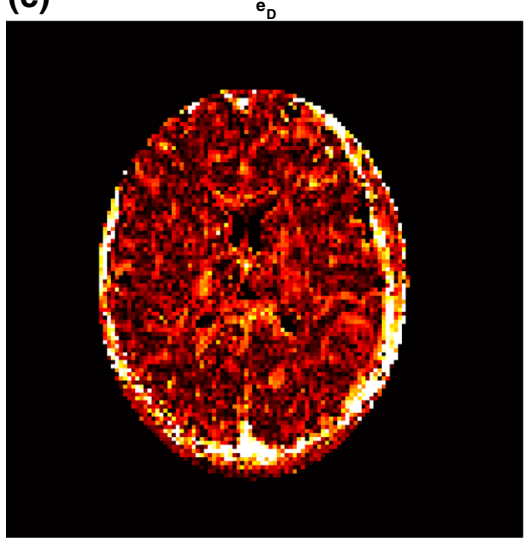

(e)

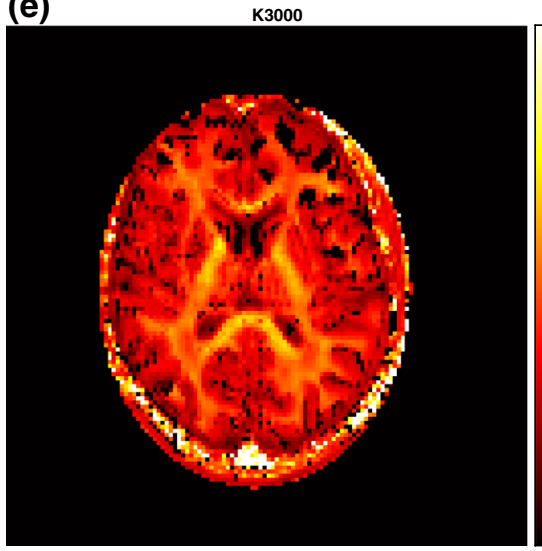

(b) $\mathrm{ADC} 3000 / \mu \mathrm{m}^{2} \mathrm{~ms}^{-1}$

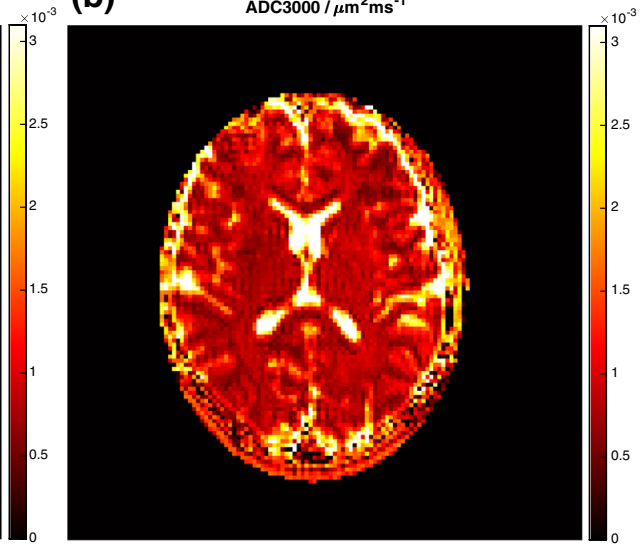

(d)

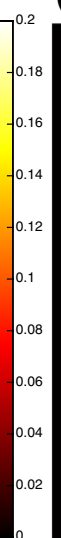

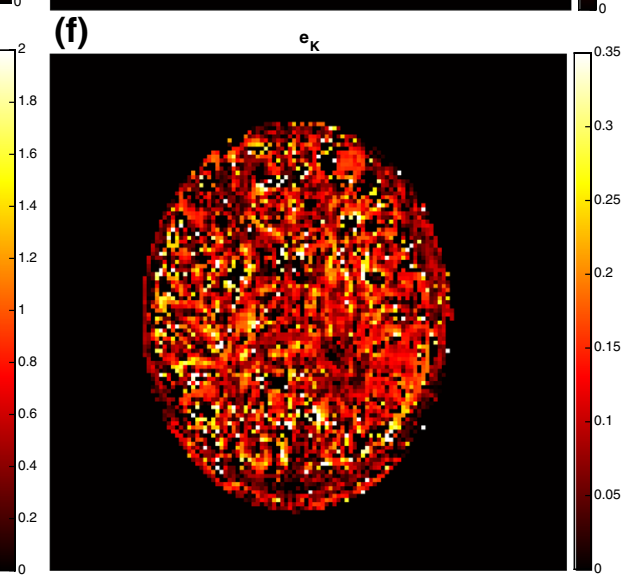

Fig. 5 Plot of ADC maps derived using maximum $b$ values of 2000 (a) and 3000 (b) $\mathrm{smm}^{-2}$, measured $e_{\mathrm{D}}$ for maximum $b$ value of $3000 \mathrm{smm}^{-2}$ (c) kurtosis maps derived using maximum $b$ values of 2000 (d) and $3000(\mathbf{e}) \mathrm{smm}^{-2}$, and measured $e_{\mathrm{K}}$ for maximum $b$ value of $3000 \mathrm{smm}^{-2}$ 
Monte Carlo simulations similar to those performed previously $[12,13]$. However, the inclusion of additional scans with $b<b_{\max }$ will tend to reduce bias and the values given by the above equations will, therefore, represent the maximum error. Finally, the equations that predict the biases did not consider bias introduced by noise in the signal. Although thermal noise has a mean value of zero, it will introduce bias due to the non-linear relationship between diffusion parameters and signal $[12,13,35]$. Hence, there is a trade-off between using high maximum $b$ values to minimize the effect of thermal noise on estimating diffusion parameters and using low maximum $b$ values to minimize the biases investigated in this study. The greater variability in estimates in Fig. 3 near the origin is probably due to the effect of thermal noise on parameter estimates. Optimization of $b$ value selection taking into account both bias and thermal noise should be the subject of future studies.

\section{Conclusion}

Despite these limitations, we believe that the above study provides valuable insight into the $b$ value dependence of diffusion parameter estimates and a useful guide to $b$ value selection. This study suggests two of methods, other than protocol standardization, of overcoming this source of protocol dependence. First, maximum $b$ value could be set to a relatively low value. This would, however, increase noise in estimates and reduce precision [12]. Alternatively, additional $b$ value scans could be acquired and higher order equations used to calculate parameters. For example, ADC could be calculated with three $b$ values using Eq. (4). This would markedly reduce bias in ADC estimates, even though substantial bias might remain in kurtosis estimates.

Acknowledgements We are thankful to Dr. Alard Roebroeck (Maastricht University) for his comments during preparation of this manuscript and for supporting presentation of the preliminary version of this work at ISMRM [36]. We are also very grateful to the National Brain Mapping Laboratory (NBML) of Tehran, Iran, and especially Hamid Reza Saligheh Rad (Ph.D., Tehran University of Medical Sciences) for allowing us to use their MRI scanner for this study.

Open Access This article is distributed under the terms of the Creative Commons Attribution 4.0 International License (http://creativecommons.org/licenses/by/4.0/), which permits unrestricted use, distribution, and reproduction in any medium, provided you give appropriate credit to the original author(s) and the source, provide a link to the Creative Commons license, and indicate if changes were made.

\section{Appendix}

\section{Derivation of $e_{\mathrm{D}}$ and $e_{\mathrm{K}}$}

Assuming $S_{1}$ and $S_{2}$ are the signal at $b_{\max } / 2$ and $b_{\max }$, respectively:

$$
\begin{gathered}
S_{1}=S_{0} e^{\frac{-D b_{\max }}{2}+\frac{K\left(D b_{\max }\right)^{2}}{24}+\frac{L\left(D b_{\max }\right)^{3}}{720}} \\
S_{2}=S_{0} e^{-D b_{\max }+\frac{K\left(D b_{\max }\right)^{2}}{6}+\frac{L\left(D b_{\max }\right)^{3}}{90}} .
\end{gathered}
$$


Then, $Q_{1}$ and $Q_{2}$ are

$$
\begin{gathered}
Q_{1}=D-\frac{b_{\max } D^{2} K}{12}-\frac{b_{\max }^{2} D^{3} L}{360} \\
Q_{2}=D-\frac{b_{\max } D^{2} K}{6}-\frac{b_{\max }^{2} D^{3} L}{90} .
\end{gathered}
$$

In addition, accordingly, $\tilde{D}$ and $\tilde{K}$ are

$$
\begin{gathered}
\tilde{D}=D+\frac{b_{\max }^{2} D^{3} L}{180} \\
\tilde{K}=\frac{D^{2} K+\frac{b_{\max } D^{3} L}{10}}{D+\frac{b_{\max }^{2} D^{3} L}{180}} .
\end{gathered}
$$

\section{Kurtosis and Ektasis for a Uniform Distribution}

For a uniform (top-hat) probability density function

$$
P(x)=\left\{\begin{array}{cc}
0 & |x|>a \\
2 / a & |x| \leq a
\end{array},\right.
$$

the $n$th moment, $\mu_{n}$, is given by

$$
\mu_{n}=\left.\frac{\mathrm{d}^{n}}{\mathrm{~d} t^{n}} g(t)\right|_{t=0},
$$

where all of the odd terms are zero because of symmetry, and $g(t)$ is the moment generating function [20]:

$$
g(t)=\frac{1}{2 a} \int_{-a}^{a} e^{t x} \mathrm{~d} x
$$

In the absence of bulk flow, odd moments (and hence the mean and skewness) are zero. The variance, $V$, kurtosis, $K$, and ektasis, $L$, are given by consecutive normalized even moments:

$$
\begin{gathered}
V=\mu_{2}=\frac{2 a}{3} \\
K=\frac{\mu_{4}}{\mu_{2}^{2}}-3=-\frac{6}{5}
\end{gathered}
$$




$$
L=\frac{\mu_{6}-15 \mu_{2} \mu_{4}-10 \mu_{3}^{2}+30 \mu_{2}^{3}}{\mu_{2}^{3}}=\frac{48}{7} .
$$

\section{References}

1. R.J. Theilmann, R. Borders, T.P. Trouard, G. Xia, E. Outwater, J. Ranger-Moore, R.J. Gillies, A. Stopeck, Neoplasia 6(6), 831-837 (2004). https://doi.org/10.1593/neo.03343

2. M. Iima, K. Yano, M. Kataoka, M. Umehana, K. Murata, S. Kanao, K. Togashi, D. Le Bihan, Investig. Radiol. 50(4), 205-211 (2015). https://doi.org/10.1097/RLI.0000000000000094

3. E. Sigmund, G. Cho, S. Kim, M. Finn, M. Moccaldi, J. Jensen, D. Sodickson, J. Goldberg, S. Formenti, L. Moy, Magn. Reson. Med. 65(5), 1437-1447 (2011)

4. N. Gilani, P. Malcolm, G. Johnson, Magn. Reson. Med. 78(1), 316-326 (2017). https://doi.org/10.1002/ mrm.26340

5. N. Gilani, P. Malcolm, G. Johnson, NMR Biomed. (2017). https://doi.org/10.1002/nbm.3782

6. M. Bester, J.H. Jensen, J.S. Babb, A. Tabesh, L. Miles, J. Herbert, R.I. Grossman, M. Inglese, Mult. Scler. J. 21(7), 935-944 (2015). https://doi.org/10.1177/1352458514556295

7. V.G. Kiselev, Diffusion MRI 2010, 152-168 (2010)

8. D.K. Jones, Diffusion MRI (Oxford University Press, Oxford, 2010)

9. E.O. Stejskal, J.E. Tanner, J. Chem. Phys. 42, 288-292 (1965)

10. J.H. Jensen, J.A. Helpern, A. Ramani, H. Lu, K. Kaczynski, Magn. Reson. Med. 53(6), 1432-1440 (2005)

11. J.H. Jensen, J.A. Helpern, NMR Biomed. 23(7), 698-710 (2010). https://doi.org/10.1002/nbm.1518

12. N. Gilani, P.N. Malcolm, G. Johnson, Appl. Magn. Reson. 47(11), 1229-1238 (2016). https://doi. org/10.1007/s00723-016-0829-X

13. N. Gilani, A.B. Rosenkrantz, P. Malcolm, G. Johnson, J. Magn. Reson. Imaging 42(4), 1072-1077 (2015). https://doi.org/10.1002/jmri.24870

14. H.C. Thoeny, F. De Keyzer, C. Boesch, R. Hermans, J. Magn. Reson. Imaging 20(5), 786-790 (2004). https://doi.org/10.1002/jmri.20196

15. N.M. deSouza, S.F. Riches, N.J. Vanas, V.A. Morgan, S.A. Ashley, C. Fisher, G.S. Payne, C. Parker, Clin. Radiol. 63(7), 774-782 (2008). https://doi.org/10.1016/j.crad.2008.02.001

16. S. Goshima, M. Kanematsu, H. Kondo, R. Yokoyama, K. Kajita, Y. Tsuge, H. Watanabe, Y. Shiratori, M. Onozuka, N. Moriyama, J. Magn. Reson. Imaging 28(3), 691-697 (2008). https://doi.org/10.1002/ jmri.21467

17. A. Chuhutin, B. Hansen, S.N. Jespersen, NMR Biomed. (2017). https://doi.org/10.1002/nbm.3777

18. S. Lanzafame, M. Giannelli, F. Garaci, R. Floris, A. Duggento, M. Guerrisi, N. Toschi, Med. Phys. 43(5), 2464-2475 (2016)

19. J.H. Jensen, M.F. Falangola, C. Hu, A. Tabesh, O. Rapalino, C. Lo, J.A. Helpern, NMR Biomed. 24(5), 452-457 (2011). https://doi.org/10.1002/nbm.1610

20. C. Grinstead, J. Snell, Introduction to Probability (American Mathematical Society, Providence, 2012)

21. D.A. Yablonskiy, G.L. Bretthorst, J.J. Ackerman, Magn. Reson. Med. 50(4), 664-669 (2003)

22. C. Liu, R. Bammer, B. Acar, M.E. Moseley, Magn. Reson. Med. 51(5), 924-937 (2004)

23. N. Gilani, P. Malcolm, G. Johnson, Magn. Reson. Med. 77(4), 1671-1677 (2017). https://doi. org/10.1002/mrm.26230

24. R.V. Mulkern, A.S. Barnes, S.J. Haker, Y.P. Hung, F.J. Rybicki, S.E. Maier, C.M. Tempany, Magn. Reson. Imaging 24(5), 563-568 (2006). https://doi.org/10.1016/j.mri.2005.12.008

25. H. Shinmoto, K. Oshio, A. Tanimoto, N. Higuchi, S. Okuda, S. Kuribayashi, R.V. Mulkern, Magn. Reson. Imaging 27(3), 355-359 (2009). https://doi.org/10.1016/j.mri.2008.07.008

26. P.J. Basser, D.K. Jones, NMR Biomed. 15(7-8), 456-467 (2002)

27. E. Fieremans, J.H. Jensen, J.A. Helpern, NeuroImage 58(1), 177-188 (2011). https://doi.org/10.1016/j. neuroimage.2011.06.006

28. H. Zhang, T. Schneider, C.A. Wheeler-Kingshott, D.C. Alexander, NeuroImage 61(4), 1000-1016 (2012). https://doi.org/10.1016/j.neuroimage.2012.03.072

29. E.S. Hui, M.M. Cheung, K.C. Chan, E.X. Wu, NeuroImage 49(3), 2366-2374 (2010). https://doi. org/10.1016/j.neuroimage.2009.10.022 
30. J. Dudink, D. Larkman, O. Kapellou, J. Boardman, J. Allsop, F. Cowan, J. Hajnal, A. Edwards, M. Rutherford, S. Counsell, Am. J. Neuroradiol. 29(10), 1966-1972 (2008)

31. R.A. Jones, S. Palasis, J.D. Grattan-Smith, J. Magn. Reson. Imaging 18(6), 665-674 (2003)

32. D.K. Jones, P.J. Basser, Magn. Reson. Med. 52(5), 979-993 (2004)

33. H. Seo, K.-H. Chang, D. Na, B. Kwon, D. Lee, Am. J. Neuroradiol. 29(3), 458-463 (2008)

34. V. Mohanty, E.T. McKinnon, J.A. Helpern, J.H. Jensen, Magn. Reson. Imaging 48, 80-88 (2018). https ://doi.org/10.1016/j.mri.2017.12.030

35. R. Fleysher, L. Fleysher, O. Gonen, Magn. Reson. Imaging 26(3), 433-435 (2008)

36. Gilani, N., H. Saligheh Rad, G. Johnson, in Proceedings of the 26th Annual Meeting of the International Society for Magnetic Resonance in Medicine (Paris, France, 2018), p. 5364 